\title{
ESTIMATES FOR THE BERGMAN AND SZEGÖ KERNELS IN CERTAIN WEAKLY PSEUDOCONVEX DOMAINS
}

\author{
ALEXANDER NAGEL, JEAN-PIERRE ROSAY, ELIAS M. STEIN \\ AND STEPHEN WAINGER
}

We announce estimates of the Bergman and Szegö kernels on domains in $\mathbf{C}^{2}$ of the form

$$
\Omega=\Omega_{P}=\left\{\left(z_{1}, z_{2}\right) \in \mathbf{C}^{2} \mid \operatorname{Im}\left(z_{2}\right)>P\left(z_{1}\right)\right\}
$$

where $P$ is a subharmonic, nonharmonic polynomial on $\mathbf{C}$. Such a domain is weakly pseudoconvex and of finite type, and this class of domains includes, for example, the case

$$
P(z)=|z|^{2 k}, \quad k \geq 1,
$$

studied in [2], [8] and [1], and the case

$$
P(z)=|z|^{8}+(15 / 7)|z|^{2} \operatorname{Re}\left(z^{6}\right),
$$

which is a domain of Kohn-Nirenberg type [5]. ${ }^{1}$

Recall that the Bergman space $B\left(\Omega_{P}\right)$ consisting of holomorphic $L^{2}$ functions on $\Omega_{P}$ is a closed subspace of the Hilbert space $L^{2}\left(\Omega_{P}\right)$. The Bergman projection is the orthogonal projection $B_{P}: L^{2}\left(\Omega_{P}\right) \rightarrow B\left(\Omega_{P}\right)$. We can write $B_{P}$ as an integral operator

$$
B_{P} f(\varsigma)=\int_{\Omega_{P}} B_{P}(\varsigma, \eta) f(\eta) d \eta
$$

where $B_{P}():, \Omega_{P} \times \Omega_{P} \rightarrow \mathbf{C}$ is the Bergman kernel for $\Omega_{P}$. If $\left\{\phi_{n}\right\}$ is a complete orthonormal basis for $B\left(\Omega_{P}\right)$ then

$$
B(\varsigma, \eta)=\sum_{n=0}^{\infty} \phi_{n}(\varsigma) \overline{\phi_{n}(\eta)}
$$

and the series converges uniformly on compact subsets of $\Omega_{P} \times \Omega_{P}$ (see, for example, [3]).

Similarly the subspace $H_{2}\left(\Omega_{P}\right)$ of $L^{2}\left(\partial \Omega_{P}\right)$ consisting of boundary values of holomorphic functions $F$ on $\Omega_{P}$ such that

$$
\|F\|_{H_{2}}^{2}=\sup _{y>0} \iint_{\mathbf{C} \times \mathbf{R}}|F(z, t+i P(z)+i y)|^{2} d z d t<\infty
$$

Received by the editors June $8,1987$.

1980 Mathematics Subject Classification (1985 Revision). Primary 32H10, 32H40, 42B20.

${ }^{1}$ The methods used here extend to cover the case of general pseudoconvex domains in $\mathbf{C}^{2}$ of finite type. 
is a closed subspace of the Hilbert space $L^{2}\left(\partial \Omega_{P}\right)$. The Szegö projection is the orthogonal projection $S_{P}: L^{2}\left(\partial \Omega_{P}\right) \rightarrow H_{2}\left(\Omega_{P}\right)$, and we can write

$$
S_{P} f(\varsigma)=\int_{\partial \Omega_{P}} S_{P}(\varsigma, \eta) f(\eta) d \sigma(\eta)
$$

where $S_{P}: \Omega \times \Omega \rightarrow \mathbf{C}$ is the Szegö kernel for the domain $\Omega_{P}$, and $d \sigma$ is the measure on $\partial \Omega$ given by $d z d t$ when we identify $\partial \Omega$ with $\mathbf{C} \times \mathbf{R}$.

The functions $B_{P}$ and $S_{P}$ are initially defined on $\Omega \times \Omega$, but they extend smoothly to $\bar{\Omega} \times \bar{\Omega} \backslash$ diagonal $(\partial \Omega)$. Our aim is to obtain sharp estimates for $B_{P}$ and $S_{P}$ and their derivatives on the set $\bar{\Omega}_{P} \times \bar{\Omega}_{P} \backslash$ diagonal $\left(\partial \Omega_{P}\right)$. These estimates will be given in terms of a natural nonisotropic metric that was introduced in [6]. We briefly recall the definition of this metric and some of its properties.

If $\Omega_{P}=\left\{\left(z_{1}, z_{2}\right) \in \mathbf{C}^{2} \mid \operatorname{Im}\left(z_{2}\right)>P\left(z_{1}\right)\right\}$, the tangential Cauchy-Riemann operator on $\partial \Omega_{P}$ is

$$
\bar{L}=\frac{\partial}{\partial \bar{z}_{1}}-2 i \frac{\partial P}{\partial \bar{z}_{1}}\left(z_{1}\right) \frac{\partial}{\partial \bar{z}_{2}}
$$

and we can view $\bar{L}$ as a complex-valued vector field on $\partial \Omega$. We write $\bar{L}=$ $\frac{1}{2}(X+i Y)$, where $X$ and $Y$ are real vector fields on $\partial \Omega$. Since $P$ is a nonharmonic polynomial, if the degree of $P$ is $m$, one can check that the vector fields $X$ and $Y$, together with their commutators of length at most $m$, span the tangent space to $\partial \Omega_{P}$ at every point. Thus $X$ and $Y$ are of Hörmander finite type $m$ (see [4]). We can now define the nonisotropic metric $\rho$ on the boundary $\partial \Omega_{P}$. Let $\varsigma_{0}, \varsigma_{1} \in \partial \Omega_{P}$. Then

$$
\begin{aligned}
\rho\left(\varsigma_{0}, \varsigma_{1}\right)= & \inf \{\delta>0 \mid \text { there exists an absolutely continuous map } \\
& \psi:[0,1] \rightarrow \partial \Omega \text { with } \psi(0)=\varsigma_{0}, \psi(1)=\varsigma_{1}, \text { and } \\
& \psi^{\prime}(t)=\alpha(t) X+\beta(t) Y \text { with }|\alpha(t)|<\delta,|\beta(t)|<\delta \\
& \text { for almost all } t \in[0,1]\} .
\end{aligned}
$$

Since $X$ and $Y$ are of finite type, it follows that $\rho\left(\varsigma_{0}, \varsigma_{1}\right)<\infty$ for any two points on $\partial \Omega_{P}$, and it is then easy to check that $\rho$ is a metric (see [7] for details).

We can obtain a better idea of the nature of this metric with the following considerations. For $z \in \mathbf{C}$, set

$$
\Lambda_{j}(z)=\sum_{\alpha+\beta=j-2}\left|\frac{\partial^{\alpha+\beta}}{\partial x^{\alpha} \partial \bar{z}^{\beta}}(\Delta P)(z)\right|, \quad 2 \leq j \leq m,
$$

and define

$$
\Lambda(z, \delta)=\sum_{j=2}^{m} \Lambda_{j}(z) \delta^{j} .
$$

We have the following estimate, which we will later use to show that our estimates for the Bergman and Szegö kernels are essentially symmetric.

LEMMA 1. There are constants $C_{1}$ and $C_{2}$ depending only on $m$ so that if $\rho(\varsigma, \eta)<\delta$, and if $\varsigma=(z, t+i P(z)), \eta=(w, s+i P(w))$, then

$$
C_{1} \leq \Lambda(z, \delta) / \Lambda(w, \delta) \leq C_{2} \text {. }
$$


We can also describe the ball $B(\varsigma, \delta)=\left\{\eta \in \partial \Omega_{P} \mid \rho(\varsigma, \eta)<\delta\right\}$ in terms of the function $\Lambda(z, \delta)$.

LEMMA 2. There are constants $C_{1}$ and $C_{2}$ depending only on $m$ so that if $P$ is a subharmonic, nonharmonic polynomial of degree at most $m$, if $\varsigma=$ $(z, t+i P(z)) \in \partial \Omega_{P}$ and if $\delta>0$, then

$$
\begin{aligned}
B\left(\varsigma, C_{1} \delta\right) & \subset\left\{\eta=(w, s+i P(w)) \in \partial \Omega_{P}|| z-w \mid<\delta\right. \text { and } \\
& \quad|s-t+2 \operatorname{Im} \tilde{P}(z, w)|<\Lambda(z, \delta)\} \\
& \subset B\left(\varsigma, C_{2} \delta\right),
\end{aligned}
$$

where

$$
\tilde{P}(z, w)=\sum_{j=1}^{m} \frac{1}{j !} \frac{\partial^{j} P}{\partial z^{j}}(z)(w-z)^{j} .
$$

Roughly, this lemma says that the ball $B(\zeta, \delta)$ is a "twisted ellipsoid" of size $\delta$ along the complex directions at $\varsigma=(z, t+i P(z))$, and of size $\Lambda(z, \delta)$ in the complementary real direction. In particular, the volume of the ball $B(\varsigma, \delta)$ on $\partial \Omega$ is

$$
\operatorname{vol}(B(\zeta, \delta)) \approx \delta^{2} \Lambda(z, \delta)
$$

(see [6 and 7] for further details).

To write down the estimates for the Bergman and Szegö kernels, we need two more pieces of notation. First, let $\Pi: \Omega_{P} \rightarrow \partial \Omega_{P}$ denote the natural projection given by

$$
\Pi(z, t+i P(z)+i y)=(z, t+i P(z)) .
$$

Second, let $h: \Omega_{P} \rightarrow(0, \infty)$ denote the height function

$$
h(z, t+i P(z)+i y)=y .
$$

We then have the following estimate for the Bergman kernel $B_{P}($,$) :$

THEOREM 1. There is a constant $C_{m}$ so that for every subharmonic nonharmonic polynomial $P$ of degree at most $m$, and for any point $(\varsigma, \eta) \in$ $\bar{\Omega}_{P} \times \bar{\Omega}_{P} \backslash$ diagonal $\left(\partial \Omega_{P}\right)$, if $\Pi(\zeta)=(z, t+i P(z))$, then

$$
\left|B_{P}(\varsigma, \eta)\right| \leq C_{m} \delta^{-2} \Lambda(z, \delta)^{-2}
$$

where

$$
\delta=\inf \{t>0 \mid \Pi(\eta) \in B(z, t), h(\varsigma)<\Lambda(z, t), h(\eta)<\Lambda(z, t)\} .
$$

Note that if both $\varsigma$ and $\eta$ are on the boundary of $\Omega_{P}$, then $\delta=\rho(\varsigma, \eta)$.

We have an analogous estimate for the Szegö kernel $S_{P}($,$) :$

THEOREM $2 .^{2} \quad$ There is a constant $C_{m}$ so that for every subharmonic nonharmonic polynomial $P$ of degree at most $m$, and for any point $(\varsigma, \eta) \in$ $\bar{\Omega}_{P} \times \bar{\Omega}_{P} \backslash$ diagonal $\left(\partial \Omega_{P}\right)$, if $\Pi(\zeta)=(z, t+i P(z))$,

$$
\left|S_{P}(\zeta, \eta)\right| \leq C_{m} \delta^{-2} \Lambda(z, \delta)^{-1}
$$

${ }^{2}$ The results for the Szegö kernel have been obtained independently by different methods by $M$. Machedon in a paper to appear in Inventiones Mathematical. 
where

$$
\delta=\inf \{t>0 \mid \Pi(\eta) \in B(z, t), h(\varsigma)<\Lambda(z, t), h(\eta)<\Lambda(z, t)\} .
$$

In particular if $(\varsigma, \eta) \in \partial \Omega_{P} \times \partial \Omega_{P}$ with $\varsigma \neq \eta$, then for another absolute constant $C_{m}^{\prime}$,

$$
\left|S_{P}(\varsigma, \eta)\right| \leq C_{m}^{\prime} \operatorname{vol}(B(\varsigma, \delta))^{-1}
$$

where $\delta=\rho(\varsigma, \eta)$.

We remark that the estimates of Theorems 1 and 2 do not appear to be symmetric in $\varsigma$ and $\eta$. However, using Lemma 1 , one can show that there are constants $C_{1}$ and $C_{2}$ depending only on $m$ so that if $(\varsigma, \eta) \in \bar{\Omega}_{P} \times \bar{\Omega}_{P} \backslash$ diagonal $\left(\partial \Omega_{P}\right)$, with $\Pi(\varsigma)=(z, t+i P(z))$, and $\Pi(\eta)=(w, s+i P(s))$, then if

$$
\delta_{1}=\inf \{t>0 \mid \Pi(\eta) \in B(z, t), h(\varsigma)<\Lambda(z, t), h(\eta)<\Lambda(z, t)\}
$$

and

$$
\delta_{2}=\inf \{s>0 \mid \Pi(\eta) \in B(w, s), h(\varsigma)<\Lambda(w, s), h(\eta)<\Lambda(w, s)\},
$$

then

$$
C_{1} \leq \delta_{1} / \delta_{2} \leq C_{2}
$$

Also, if $(\varsigma, \eta) \in \partial \Omega_{P} \times \partial \Omega_{P}$ and $\rho(\varsigma, \eta)=\delta$ then

$$
C_{1} \leq \operatorname{Vol}(B(\varsigma, \delta)) / \operatorname{Vol}(B(\eta, \delta)) \leq C_{2} .
$$

We also obtain estimates for derivatives of the kernels $B_{P}$ and $S_{P}$. We have

THEOREM 3. For each integer $k \geq 0$ there is a constant $C_{k, m}$ depending on $k$ and $m$ so that if $P$ is a subharmonic nonharmonic polynomial of degree at most $m$, if $(\varsigma, \eta) \in \bar{\Omega}_{P} \times \bar{\Omega}_{P} \backslash$ diagonal $\left(\partial \Omega_{P}\right)$, if $\Pi(\varsigma)=(z, t+i P(z))$, and if $\delta=\inf \{t>0 \mid \Pi(\eta) \in B(z, t), h(\varsigma)<\Lambda(z, t), h(\eta)<\Lambda(z, t)\}$, then

(a) $\left|L_{i_{1}} L_{i_{2}} \cdots L_{i_{k}} B_{P}(\zeta, \eta)\right| \leq C_{k, m} \delta^{-k-2} \Lambda(z, \delta)^{-2}$,

(b) $\left|L_{i_{1}} L_{i_{2}} \cdots L_{i_{k}} S_{P}(\zeta, \eta)\right| \leq C_{k, m} \delta^{-k-2} \Lambda(z, \delta)^{-1}$,

where each $L_{i_{j}}$ denotes either the tangential Cauchy-Riemann operator $\bar{L}$ or its conjugate $L$, and the derivatives are taken either in the $\varsigma$ variable or the $\eta$ variable. In particular, if $(\varsigma, \eta) \in \partial \Omega_{P} \times \partial \Omega_{P}$ and $\rho(\varsigma, \eta)=\delta$, then

$$
\left|L_{i_{1}} L_{i_{2}} \cdots L_{i_{k}} S_{P}(\varsigma, \eta)\right| \leq C_{k, m} \delta^{-k} \operatorname{Vol}(B(\varsigma, \delta))^{-1} .
$$

Note that on the boundary all derivatives can be written as commutators of $L$ and $\bar{L}$, and so Theorem 3 shows how to control all derivatives on the boundary. One can now apply fairly standard singular integral and fractional integration machinery to prove various regularity properties of the Bergman and Szegö projections. Further discussion and complete proofs will appear in a forthcoming paper. 


\section{REFERENCES}

1. K. P. Diaz, The Szegö kernel as a singular integral kernel in a weakly pseudoconvex domain, Dissertation, Princeton Univ., 1986.

2. P. C. Greiner and E. M. Stein, On the solvability of some differential operators of type $\square_{b}$, Proc. Conf. (Cortona, Italy, 1976-1977), Scuola Normale Superiore, Pisa, pp. 106-165. 1962.

3. S. Helgason, Differential geometry and symmetric spaces, Academic Press, New York,

4. L. Hörmander, Hypoelliptic second order differential equations, Acta Math. 119 (1967), 147-171.

5. J. J. Kohn and L. Nirenberg, A pseudo-convex domain not admitting a holomorphic support function, Math. Ann. 201 (1973), 265-268.

6. A. Nagel, E. M. Stein and S. Wainger, Boundary behavior of functions holomorphic in domains of finite type, Proc. Nat. Acad. Sci. U.S.A. 78 (1981), 6596-6599.

7. (1985), 103-147.

8. A. Bonami and N. Lohové, Projecteurs de Bergman et Szegõ pour une classe de domaines faiblement pseudo-convexes et estimations $L^{p}$, Compositio Math. 46 (1982), 159-226.

Department of MAthematics, UNiversity of Wisconsin, Madison, WisCONSIN 53706 (Address of A. Nagel, J.-P. Rosay and S. Wainger)

Department of Mathematics, Princeton University, Princeton, New JERSEY 08544 
